\title{
Team Based Approach to Psychiatric Treatment: A Model that Works from the Patient's Viewpoint
}

\author{
Cusack PT* \\ Independent Researcher, Canada
}

*Corresponding author: Paul T E Cusack, Independent Researcher, 23 Park Ave, Saint John NB, Canada, Tel: (506) 652-6350; Email: St-michael@hotmail.com

\section{Research Article}

Volume 2 Issue 2

Received Date: March 13, 2018

Published Date: April 09, 2018

\begin{abstract}
A patient's resent observation of the team-based approach to psychiatric treatment in a socialized setting at one hospital. It was found that the team-based approach has many advantages and some disadvantages but generally works well, although it could be improved upon if managed properly by Nurses and Psychiatrists. Recommendations are suggested to improve on this model of treatment.
\end{abstract}

Keywords: Team-based psychiatric treatment; Psychiatric Nursing; Socialization

Abbreviations: ECT: Electroconvulsive Therapy; OT: Occupational Therapist; SD: Spiritual Director; SW: Social worker.

\section{Introduction}

The purpose of this paper is not to provide in depth research in Nursing, but rather to prove the patient's view of team based psychiatric nursing. It is admitted that a through comparison with other models of nursing /psychiatric may not be done here as the author is not privy to other models. Instead, a simple observation is made in an afford to provide a start to an evaluation on various treatment models.

\section{Materials/ Methods}

The Author was hospitalized at the Saint John Regional Hospital on Aug 3, 2017 -Oct 6, 2017 for an undisclosed reason. He was admitted to 4D North- the Psychiatric Ward where he hadn't been for 20 years. In 1997 and 1998, he was hospitalized for a couple of months each time. The hospital authorities had made changes in the way patients were treated for mental illness in the psychiatry ward. The new treatment model necessitated changes in the physical layout of the floor to allow for team-based treatment. See Figure 1 for the latest layout for 4D north. 


\section{Nursing \& Healthcare International Journal}

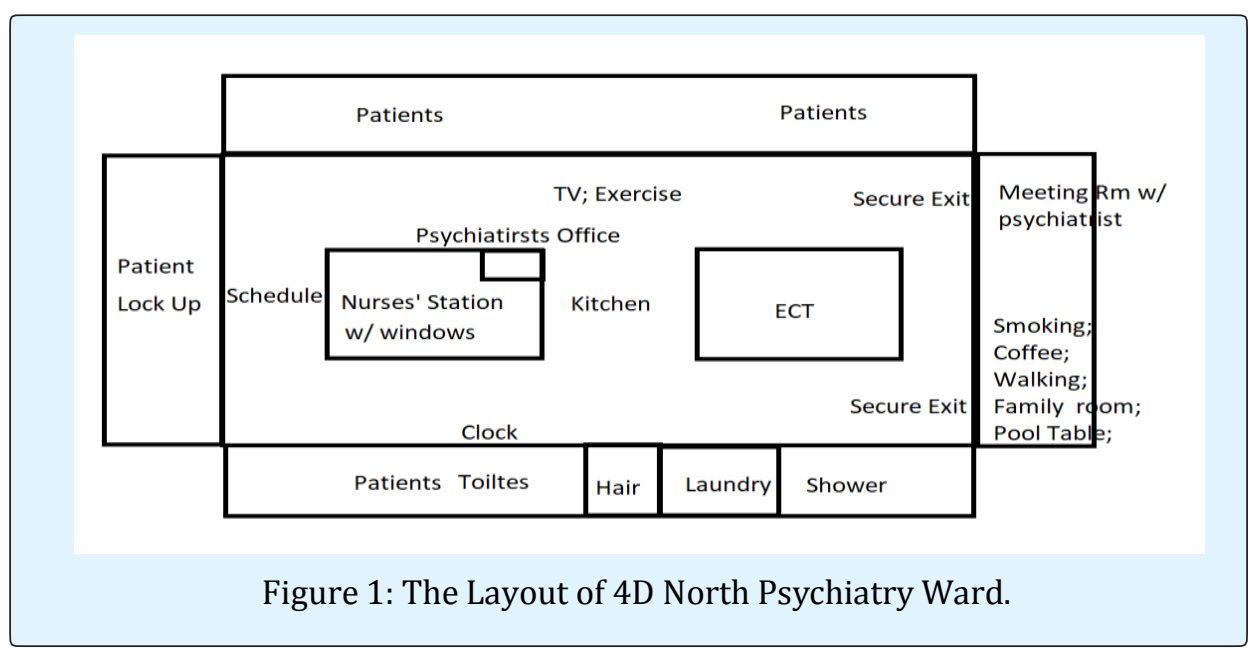

Whereas previously the floor had an Observation Unit, and a distant dining room, the new treatment facility had a kitchen -dining room as the central to the patients. The psychiatrists had a booth looking on the main dining area and there was a TV room which also allowed for exercise space and puzzle building.

Each patient was assigned a room with one roommate. The room had one toilet room. Each room had large windows and two beds and four visitor's chairs plus locker space. These rooms surrounded the main gathering socialization area. At the far end of the floor were several individual lock-ups for Code White (Violent) patient's, usually those lately admitted. In addition, there is a slower; laundry; and an electroconvulsive therapy (ECT) room. The entire area was secured by electronic locks that required a pass for off unit privileges.

After admission to the ward, generally though the Lockup, and treatment for a code white (violent behaviour), the patient is discharged to his or her room on the floor to be shared with a roommate. A Psychiatrist is assigned to the newly admitted patient. The RN's are the second person the patient encounters after being admitted by a psychiatrist in the ER. Medications may be administered immediately after admission.

After the patient recovers from the episode, he or she starts receiving meals regularly three times per day (7:30; $11: 30 ; 4: 30$ ) the meals are brought in to the floor on a dolly and are self served. The patient selects where and with whom he or she will eat -four to a table.

After several days, the patient is encouraged to participate in activities designed to help the patient learn new skills and become regular in his or her schedule. Visitors are permitted. A telephone is available for outside calls.

Gradually, the patient is introduced to the tram of professionals and staff he or she will be working with. They included those in the following (Figure 2). There is room for approximately 26 patients' and about the same number of RN's.

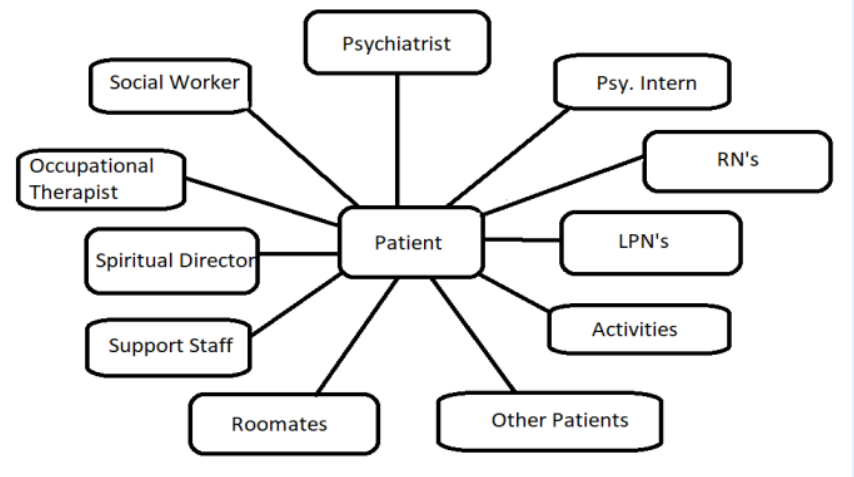

Figure 2: The Team. 


\section{Nursing \& Healthcare International Journal}

The Psychiatrists are gathered in a lookout on the Dining area and the TV room. There are Medical Doctor Interns training to be psychiatrists who shadow a practicing psychiatrist. The RN's and LPN gather in the nursing station. There is also space for the OT's and the Social workers.

The patients are encouraged by the structure of the layout to interact with other patients. When not in their room, the patient is usually in the kitchen playing games, or watching TV. Many off-unit passes are dispensed each day so that the patient can leave the floor with permission. Medications are dispensed as prescribed by a Med RN for a dolly cart.

There are also activities/help sessions run by an activity coordinator and involve things such as Art Therapy; BINGO; Pool Table; Cooking; Exercise. The idea is to keep the patient engaged with the program while leering new skills. Socialization is the undercurrent. The patient is encouraged to stay connected with his or her family. A telephone is provided as well as visitors are permitted. Usually, off unit privileges are granted and allow a patient to make a visit with a family member off hospital grounds.

There are three other support workers part of the team headed by the psychiatrist. They are the Occupational Therapist (OT); the Spiritual Director (SD); and the Social worker (SW). The OT meets with the patient twice a week and discusses things such as having a healthy diet and having a plan for the patient's life. The SD meets in groups twice per week wherein patients share what they care to about how we treat oneself. The SW meets periodically to forge a plan regarding the living situation after discharge. He turns the case over to another SW who has periodic meeting after discharge. This is all the players in the team except for the cleaners who clean the rooms and living spaces every day.

\section{The Nurses}

The Team on 4D North has 24-7 nursing. There are the two levels of nursing care: the RN's and the LPN's. They support the Team by providing daily care to the psychiatric patients. They are assigned each day to a few patients, beginning the shirt with an introduction; mood inquiry; issues; blood pressure and temperature. Weight is collect from every patient once per week. Any changes in the patients' condition is brought up and discussed with the team headed by the psychiatrist and supported by the support workers (RN; LPN; SW; SD: OT)
The RN's have lots of opportunity to exercise their nursing skills. A list of adjectives witnessed by the author, include, nurses who were:

Confident; stable; gentle; professional; careful; upbeat; uptight; burnt out; argumentative; aggressive; kind; nervous; communicative; trouble shooting; interested; and friendly.

\section{The Patients}

They were approximately the same number of nurses as patients. The patients were interred for a variety of reasons, including:

Suicide; Depression; Schizophrenia; Bipolar; Gender Dysphoria; Anxiety; Sheltered; Poor Childrearing; Substance Abuse; Attention seeking; Divorce Repercussions; Syncytium; Racal Hatred; Physical Trauma; and Sexual Disfunction; Delusions of Grandeur; Deviant.

The length of stay ranged for 48 hours to 6 months. One patient was reassigned to Ridgewood; the addictions hospital for the region.

\section{Results/ Discussion}

\section{Advantages}

There are advantages to treating psychiatry patient in a team-based approach. One is the degree of socialization. The patient is immediately immersed at his or her own pace to activities such as common meals, activities, or leisure. This fallows the patient to come out of himself free from ruminating.

The nurses get a chance to act as confidante. This way, the nurses who have the time the psychiatrist doesn't, can really get to know the patient and their issues. Bedside manner present in most of the nurses is utilized.

With the potential for conflict, especially in such a setting, the nurse is called upon to be brave to face a violent patient. Depending upon the nature of the nurse, it can be good or bad.

Because nurses get to know the patient so well, there is opportunity to show compassion. The nurses are prewet in the meetings, with the psychiatrist generally. The nurse leans more about the patient in depth and can report on it.

RN's are called upon to dispense the medication to the patients. This is an essential component of the patients' treatment, of course, and must be done accurately. The patient will sometimes request additional medication, 


\section{Nursing \& Healthcare International Journal}

such as Ativan. The nurses don't prescribe this medication; but rather call upon the psychiatrist. This could result in a delay that might be painful for the patient.

Another advantage to the team-based approach is that if one of the team members is disabled, the treatment can go on without him/her for as long as it takes to get a replacement.

Multi-professional meeting allows for protection of booth the patient and professional from unwanted sexual misconduct.

Finally, listening to the patient is also crucial to the patients' well being and treatment. It is important to remember, in patient care, one size does not fit all. The Team approach can work marvellously when the patients are listened to.

\section{Disadvantages}

The main disadvantage to team-based treatments is the stress caused by the intense socialization. This particularly affects the introverts. The only refuge is the patient's room which he shares also with one other roommate, or even with three other roommates. The patients who are teamed up as roommates are an important decision. Because of the socialization of the set up, tension can result. The only place to flee is this locked up situation is to the patient's room. I the is no peace there, then a situation erupts.

Code white or violent person is damaging to the sense of well being. It is not conducive to healing of the mind but can't seem to be avoided. It is stress full for both the patient and the nurses and psychiatrist.

One outlet was enjoyed by patients was the participation in artwork. Others enjoyed movie or TV watching. Still others enjoy music. The music can get out of hand since it affects all who are within listening distance.

With the younger men and women, there are sexual tensions which can lead to problems. Some of the younger girls were assaulted. One patient can change the flavour of the cohesion. There were also occasions of physical conflict between the younger men.

The author also witnessed food hogging. Milk and juice containers as well as ice cream are put in the community refrigerator. This leads to opportunities for taking more than one's fair share.

Another opportunity for conflict between the patients is in TV hogging. Not everyone wants to watch the same show at the same time. Some patients go to the extent of hiding the remote-control device for the TV/ DVD.

Yet another point of conflict comes in the shared laundry. Patients do their own laundry and have the potential to forget that their laundry is finished. They fail to retrieve it and thus end up with the clothes spread over the laundry.

Off Unit privileges are another potential for problems. One patient went to the extend to attempt suicide by walking to the nearest bridge. The Police recovered him just before the patient was about to jump.

Off Unit privileges are necessary for those who smoke since smoking is banned on Hospital property. Bumming cigarettes can be an issue. The policy is that patients shouldn't be dealing with nicotine cravings while recovering from mental illness as well.

Violence can be a problem. One young male patient was punching other patients in the face. This leaves the award feeling like a prison where only the tough survive. The nurses showed great bravery is dealing with violent patients.

One patient had a cellular phone which was banned from the floor. It is called a "Not spot". No cellular telephone allowed. Hiding of the phone cause justified for a search and seizure.

Despite a computer being available, it was not permitted to be used by the patients. A patient loses contact with business associates with on the floor which could be 2 months or longer.

All in all, nurses are closer to the patients that the psychiatrist. Their reporting on the daily mood and issues of the patient is critical to the patient's recovery and discharge. Nurses have a different patient group to look after every day.

\section{Conclusion/ Recommendations}

The Team based approach is a valid one for treating a variety of psychiatric illness, when combined with good professional care and medications. 


\section{Nursing \& Healthcare International Journal}

The intense socialization must be applied gradually, especially for introverts. Although there are potential for conflicts between patients, it is recommended that they be permitted so the patients can get along with each other, modeling the outside world. Care should be taken in issuing off unit privileges. They should be immediately restricted if the rules are broken. Close monitoring of violent patients is recommended to keep the peace on the unit. One or two patients can increase the conflicts and the stress for the whole floor. To avoid burnout with the RN's, there should be at least 3 days off consequently to recoup form the stresses involved. Pay scales for all the employees and professionals should be more than adequate to compensate for the demands of the profession of nursing the mentally ill and to avoid burnout among nurses. There should be a good mix between male and female nurses and between age groups since the patients are mixed. The Nurses should use the Code-White sparingly to control the patients. Family and/or community support are essential for the recovering, discharged patient. 